\title{
A review of becaplermin gel in the treatment of diabetic neuropathic foot ulcers
}

\section{Robert C Fang \\ Robert D Galiano}

Division of Plastic and Reconstructive Surgery and Wound Healing Research Laboratory, Department of Surgery, Northwestern University Feinberg School of Medicine, Chicago, IL, USA
Correspondence: Robert D Galiano Division of Plastic and Reconstructive Surgery, Department of Surgery, Northwestern University Feinberg School of Medicine, Galter Pavilion 19-250, 675

N. St. Clair Street, Chicago, IL 606I I, USA

Tel + I 3I26956022

Fax + I 312695 5672

Email rgaliano@nmh.org

\begin{abstract}
Diabetic neuropathic foot ulcers represent a serious health care burden to patients and to society. While the management of chronic diabetic foot ulcers has improved in recent years, it remains a frustrating problem for a variety of clinicians. This review examines the scientific underpinnings supporting the use of becaplermin (Regranex ${ }^{\circledR}$; Ortho-McNeil Pharmaceutical, Raritan, NJ), or recombinant human platelet-derived growth factor (rhPDGF-BB), in diabetic forefoot wounds. An emphasis is placed upon proper medical and surgical care of diabetic foot wounds, as multiple studies have demonstrated that the success of this growth factor in accelerating healing is ultimately dependent on proper ulcer care. A focus on the costeffectiveness of this form of therapy in the treatment of diabetic foot ulcers is also outlined.
\end{abstract}

Keywords: becaplermin, diabetes, foot ulcer, growth factor

\section{Introduction}

Diabetes mellitus leads to a host of serious complications in multiple organ systems. Lower extremity ulcers represent a serious health care burden in terms of harm to a patient's health and cost to society. This burden is expanding tremendously, as the incidence of diabetes in industrialized as well as developing countries is growing in epidemic proportions. The annual incidence of foot ulcers in diabetic patients in the United States is estimated to be $2 \%$ of the greater than 20 million people affected by this disease, and this number rises to $57.5 \%$ among those with peripheral neuropathy. Eventually, $15 \%-25 \%$ of all people with diabetes will develop a foot ulcer at some point in their lifetime. The management of foot ulcers and their associated morbidities is a leading cause of hospitalizations, and the occurrence of a foot ulcer significantly increases the risk of an amputation in diabetic patients. In fact, approximately $85 \%$ of all amputations for diabetic limbs were preceded by ulcers, culminating in 60,000-80,000 amputations performed yearly secondary to the failure of such wounds to heal. Indeed, most lower extremity amputations are performed in diabetic patients. The risk of death for a diabetic person who ends up with a below-knee amputation is $50 \%$ over 5 years. Much of this increased risk is attributable to the increased work of ambulating with a prosthetic limb and the resulting myocardial strain in these patients, most of whom have cardiovascular disease (Centers for Disease Control and Prevention 2005; Cowie et al 2006).

It is sobering to think that most of these problems are avoidable. Most ulcers can be prevented with intense patient education, proper orthotic use, and vigilant foot care. Once diagnosed, most forefoot ulcers are curable without major surgical intervention. The onset of a mal perforant ulcer should mobilize a set of resources consisting of adherence to good wound care, offloading, and dedicated monitoring. These resources may include growth factor therapy in select circumstances. However, the proper use of biologics is only undertaken by understanding the principles of diabetic foot care and wound healing and will only be successful when other impediments to healing are addressed. 
This review will examine the scientific underpinnings supporting the use of becaplermin (Regranex ${ }^{\circledR}$; OrthoMcNeil Pharmaceutical, Raritan, NJ), or recombinant human platelet-derived growth factor (rhPDGF-BB), in diabetic forefoot wounds. As of this writing, becaplermin is the only growth factor approved by the United States Food and Drug Administration (FDA) for use in diabetic forefoot ulcers. We will review the basic tenets of proper medical and surgical care of diabetic foot wounds, as the success of this growth factor in accelerating healing is so intimately dependent on proper ulcer care. We will also outline our indications for the use of becaplermin, with a focus on the cost-effectiveness of this form of therapy.

\section{Pathogenesis of diabetic foot ulcers}

A variety of factors contribute to the pathogenesis of foot ulcers in diabetic patients, including peripheral neuropathy, ischemia, trauma, and infection (Table 1). Diabetic foot ulcers share several causative factors with other types of chronic wounds, including local tissue hypoxia, repetitive ischemia-reperfusion (IR) injury, bacterial colonization, and altered cellular and systemic stress responses. However, the diabetic foot ulcer is unique in several regards (Medina et al 2005).

The most significant factor unique to the development of ulceration is peripheral neuropathy (symmetrical distal polyneuropathy), which affects all nerve fiber types in the foot. Neuropathy of the motor fibers leads to weakness, muscle atrophy, and paresis. The intrinsic muscles in the foot are disproportionately affected, while the extrinsic muscles (represented by the long flexor and extensor tendons originating in the calf) retain most of their strength and mechanical power. This upsets the delicate balance inherent to the function of the intrinsic foot muscles so important for normal gait pressure patterns and results in the typical posture of a diabetic foot. In severe cases this leads to a "Charcot foot" with a claw-type posturing and most importantly to the transfer of most of the pressure during the gait cycle to the area underneath the metatarsal heads. The pressure placed under the metatarsal heads is usually alleviated by a fat pad; interestingly, this fat pad is often either lost or displaced in the diabetic foot. Concomitantly, a sensory neuropathy abolishes the protective function of the pain fibers. The patient is unable to sense minor trauma to the foot (for example, a small pebble under a sock or friction from a poorly-fitted shoe). As trauma is additive throughout the several thousand steps taken daily, even a small insult can result in overt cumulative injury. Finally, autonomic
Table I A variety of factors contribute to the pathogenesis of a diabetic foot ulcer

Peripheral neuropathy

Ischemia

Trauma

Infection

Local tissue hypoxia

Ischemia-reperfusion injury

Bacterial colonization

Altered cellular and systemic stress responses

nerve fiber dysfunction causes altered vasomotor control of the microcirculation and adversely affects inflammatory cascades, as well as causing anhidrosis of the plantar foot. The dry skin can lead to fissuring (which serves as an entry portal for bacteria) and aggravates plantar hyperkeratosis at areas of friction. Together, these deficiencies promote abnormal biomechanics during weight-bearing and ambulation that lead to unrecognized repetitive trauma. The skin at sites such as the plantar surface beneath the metatarsal heads or pressure points from poorly fitting shoes becomes prone to an injury that progresses into a chronic wound (Boulton et al 2004; Cavanagh et al 2005; Wieman 2005).

Local tissue hypoxia is also a major component of many diabetic foot ulcers. Regional tissue ischemia resulting from peripheral vascular disease complicates a large number of the wounds. Diabetic patients develop a stereotypical pattern of lower extremity atherosclerotic lesions in a more peripheral distribution when compared to non-diabetic patients. However, even limbs free from overt arterial insufficiency may suffer from a number of abnormalities in the microcirculation that contribute to local tissue hypoxia. Diabetic microangiopathy is characterized by distinct functional abnormalities and aberrant angiogenesis, resulting in impaired vasodilation and physiological responses to hypoxia (LoGerfo et al 1984; Rayman et al 1986; Rayman et al 1995; Jaap et al 1996; Martin et al 2003).

The repetitive nature of the trauma occurring at the vulnerable pressure points on the diabetic foot likely causes cyclical bouts of cutaneous ischemia-reperfusion (IR) injury. The effects of IR injury have been studied extensively in other organs such as the heart and brain. However, the hypothesis that cutaneous IR injury plays a role in chronic wounds has only recently been explored. On a molecular level, the injury process is initiated when adenosine triphosphate (ATP) production is reduced during ischemia, leading to cellular events that result in leukocyte activation, oxidative stress, and microvascular dysfunction upon reperfusion. Similar 
to other chronic wounds, both reperfusion and the additive effects of repetitive IR cycles can escalate the amount of injury in diabetic ulcers. With already altered mediators of IR injury, diabetic patients appear to experience a further delay in wound healing (Mustoe et al 2006).

Although not strictly a precipitating factor in diabetic foot ulcer development, an infection can quickly become established in the foot with the initial injury serving as an ingress for bacteria past the barrier defenses of intact skin. An impaired leukocyte response allows rapid spread of the infectious process, leading to added morbidity with further impaired healing, propagation of tissue damage, increased hospitalization, and ultimately amputation in a significant proportion of cases (Boulton et al 2004; Falanga 2005).

\section{Molecular and metabolic derangements of healing in diabetic patients}

The molecular pathways that culminate in wound healing deficiencies in diabetic individuals have been the focus of numerous studies. From a biochemical approach, metabolic mechanisms have been demonstrated to result in increased oxidative stress in cell types susceptible to hyperglycemia. Brownlee (2005) has recently laid out a unifying hypothesis that places the overproduction of reactive oxygen species (ROS) at the center of the four main biochemical derangements present in hyperglycemia: (1) overstimulation of the protein kinase $\mathrm{C}$ (PKC) signaling pathway, (2) overproduction and accumulation of advanced glycation end products (AGES), (3) increased accumulation of the sorbitol pathway, and (4) increased flux through the hexosamine pathway (Figure 1).

These molecular derangements have demonstrable clinical implications. For example, sorbitol accumulation in nerves and their vasa nervosum is thought to contribute to the polyneuropathy present in diabetic patients (Engerman et al 1994; Gabbay et al 1966). The increase in PKC can result in alterations in microvascular blood flow, in part through deranged nitric oxide expression, hexosamine pathway abnormalities, vessel matrix thickening from transforming growth factor$\beta$ (TGF- $\beta$ ) signalling, and increased inflammation through nuclear factor-kappa B (NF-кB) cascades (Ishii et al 1996; Koya et al 1998). Finally, AGES accumulate in tissues with slow turnover, such as tendons and skin, causing increased stiffness and thickness. By actions through their receptor (RAGE), they can also induce a pro-inflammatory phenotype (Hammes et al 1991; Schmidt et al 1995; Brownlee 2005).

Cells such as the fibroblasts and macrophages display both dysfunctional expression of and responses to many growth factors and cytokines. Affected signalling molecules include platelet-derived growth factor (PDGF), TGF- $\beta$, vascular endothelial growth factor (VEGF), tumor necrosis factor- $\alpha$ (TNF- $\alpha)$, interleukin-1 (IL-1), matrix metalloproteinases (MMP's), among others (Bennett et al 1993; Cross et al 2003). It is therefore not surprising that most growth factor monotherapies have proven to be ineffective in healing diabetic ulcers (Robson et al 1998). PDGF may be an exception, since it has pleiotrophic effects on a variety of cell types (Pierce et al 1991). The induction of a senescent-like phenotype has also been proposed as a theory for the impaired activities of these cells, likely resulting from the chronic

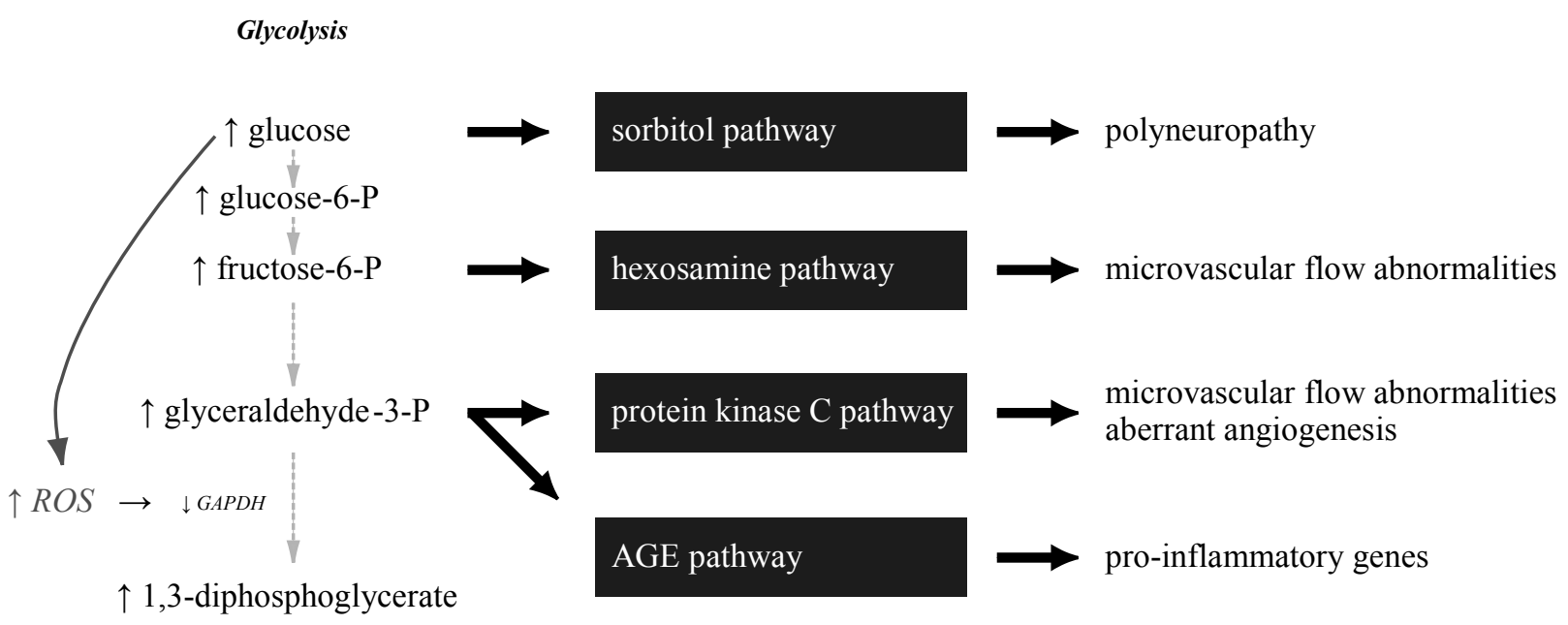

Figure I Hyperglycemia induces biochemical derangements in susceptible cells that increases free radical production, activates 4 pathways of cellular damage, and culminates in the complications that are seen in diabetic individuals. 
oxidative stress induced by hyperglycemia or by age. Growth factor therapy may "rescue" these senescent cells by activating them to enter the cell cycle or by stimulating receptive cells in a milieu that is relatively deficient in growth factors (Blazer et al 2002; Mustoe et al 2006).

\section{Current management strategies for diabetic foot ulcers}

The overall management of chronic diabetic foot ulcers has improved as a result of national and international health care efforts to assemble scientific data, foster expert discussion, and develop consensus opinions and recommendations (Apelqvist et al 2000; Frykberg et al 2006; Steed et al 2006). The care of diabetic foot ulcers is ideally rendered through a multi-disciplinary approach. The value of prevention cannot be over-emphasized. Responsible glycemic control, appropriate footwear, good foot hygiene and care, appropriate management of comorbidities, and disease education are basic elements should be incorporated into the life-style of all diabetic patients. Some authors suggest that these practices can decrease the occurrence of foot ulcers by up to $50 \%$ (Boulton et al 2004; Rathur et al 2007).

The vascular, neurological, and infectious states of the limb form the tripod supporting all subsequent management of the diabetic foot. Evaluation of the vasculature begins with the assessment of pulses and noninvasive evaluation of the peripheral circulation. Appropriate consultation of a vascular surgeon can then be undertaken for possible revascularization or alternative interventions to optimize blood supply and perfusion. Neuropathy can be evaluated with methods such as sensory testing with a nylon monofilament (Semmes-Weinstein 5.07/10 g monofilament) or biothesiometer, along with the physical exam (Steed et al 2006).

Infection must be treated aggressively. Early sharp debridement should be performed to remove all necrotic tissue, followed by a deep tissue culture. In all cases of local infection, the possibility of osteomyelitis should always be entertained. The wound should be probed to determine whether it tracks into bone or tendon. If so, broad spectrum antibiotics and further surgical debridement are necessary. Frequent and adequate sharp debridement on a regular basis has been shown to decrease wound healing times. Specifically, debridement accomplishes three functions: (1) it clears devitalized tissue and biofilm; (2) it removes callus, which serves only to increase pressure; (3) it converts a chronic, stagnant wound into an "acute" wound, which is ready to progress through the normal phases of wound healing. The most important principle of local wound care after achieving a clean wound bed consists of maintaining a clean, moist healing environment. Dressing selection need not be fancy but should match the wound characteristics and be tailored to the wound exudate level to achieve the ideal moist conditions on the wound surface (Wieman 2005; Brem et al 2006; Rathur et al 2007).

The principle of off-loading is crucial to eliminating the trauma that not only precipitated the diabetic foot ulcer but may subsequently prevent its healing. This aspect of the wound care is perhaps one of the most difficult for patients to adhere. The consequences of a lapse in adequate off-loading that reinitiates IR injury are not trivial and can undo weeks of healing progress. Indeed, this is the most common cause of recidivism in a healed ulcer. Modalities of off-loading, including orthotics, casts, boots, and foam dressings, must be individualized to the patient. The total-contact cast (TCC) represents the current gold-standard for off-loading. Remarkably, most ulcers will eventually heal solely with the use of a TCC and proper conservative wound care. Unfortunately, all forms of off-loading require large expenditures in terms of supplies and personnel, as well as a firm commitment to their proper use by the patient (Mustoe et al 2006; Wu et al 2006; Rathur et al 2007).

Adjunctive and alternative therapies are generally considered if the implementation of standard strategies has not resulted in acceptable healing of the foot ulcer. Weekly or biweekly measurements are important in this regard, as they will identify as early as possible those wounds that have stalled or have fallen off the trajectory of expected healing (Steed et al 2006). Becaplermin, tissue-engineered skin substitutes, low-frequency ultrasound, negative-pressure therapy (V.A.C.; KCI, Inc., San Antonio, Texas), and hyperbaric oxygen therapy (HBOT) have all had promising results in clinical trials and are being utilized more often for difficult non-healing foot ulcers. Other interventions such as electrical stimulation, light-emitting diodes, and hydrotherapy do not have clear supporting data at this time (Boulton et al 2004; Steed 2006).

Surgical procedures have been advocated for selected cases. An amputation may sometimes be more desirable instead of a prolonged effort at limb salvage, particularly in patients with unreconstructable vascular supply and active infection or gangrene. However, efforts to preserve a limb are generally worthwhile, due to the overall deleterious effects of leg amputations in these patients. Metatarsal head resections can be designed to be beneficial in relieving pressure points on the plantar foot surface. More proximal hindfoot amputations, despite their unappealing cosmetic 
appearance and the relative inexperience of many pedorthists in designing prostheses for such stumps, are nevertheless useful in diabetic patients to maintain ambulatory ability with minimal increases in energy expenditure (Waters et al 1976; Pinzur et al 1992; Stone et al 2005). Reconstructive plastic surgical procedures are extremely useful for covering areas of exposed bone or tendon, for larger defects, and for defects of the midfoot and hindfoot (Attinger et al 2002). Some have advocated Achilles tendon lengthening to reduce the pressure on forefoot plantar ulcers (Mueller et al 2003; Steed et al 2006). Other procedures such as a tarsal tunnel release have shown promise in cases of distal polyneuropathy, with evidence of superimposed nerve compression, but these interventions require further evaluation for efficacy at this time (Valdivia et al 2005; Chaudhry et al 2006).

\section{Growth factor therapy for chronic wounds}

Despite adequate care of diabetic foot ulcers, many neuropathic ulcers on non-ischemic limbs heal slowly or fail to heal, increasing the risk of infections and subsequent amputations. Surgical options for closure may be limited due to comorbidities in these often ill patients. The need for new modalities has spurred the application of molecular therapeutics, in particular growth factors, to the care of chronic wounds. The use of growth factors as vulnerary agents in preclinical models arose soon after their discovery. In the 1980s, PDGF became the first growth factor that was isolated and purified to homogeneity. Concurrently, other studies had demonstrated the integral roles of factors such as PDGF, TGF- $\beta$, and fibroblast growth factor (FGF) in wound healing. Clinical studies, meanwhile, had shown that levels of several important growth factors were significantly decreased in chronic wounds when compared to acute surgical wounds. With these novel findings regarding the cell biology of chronic wounds, the rationale that the replacement or addition of growth factors to the wound milieu would lead to a positive effect on wound healing certainly appeared promising (Mustoe et al 1991; Pierce et al 1988, 1991).

However, with the exception of PDGF, growth factor monotherapies for chronic wounds have not developed into successful therapeutics. One fundamental problem involves the local protease-rich environment of a chronic wound, which has been shown to actively degrade and inactivate most growth factors. This finding may explain in part the altered levels of growth factors in chronic wounds, but it also suggests that topical therapeutic applications probably allow only a brief suboptimal exposure of the wound to the growth factor unless the protease-rich, pro-inflammatory wound environment is first addressed (Robson et al 1998; Cross et al 2003).

PDGF was first identified as a mitogen for fibroblasts and smooth muscle cells (SMC's). The growth factor was subsequently found to also induce chemotaxis for these cells and other cells that enter the healing wound, such as neutrophils and monocytes. It was initially isolated from platelets but was later shown to be produced by most of the activated cells present in the wound milieu. One principal effect of PDGF on wound healing is the stimulation of extracellular matrix (ECM) synthesis and collagen production. Once activated, fibroblasts produce provisional matrix components such hyaluronic acid and fibronectin. Eventually, the synthesis of type I collagen leads to the development of wound strength. PDGF is also known to activate several intracellular signaling cascades through different receptors, leading to its pleiotropic effects in a wound (Pierce et al 1988; Pierce et al 1991; Ross et al 1974).

These effects of PDGF do not remain constant but differ over the course of the wound healing process (Figure 2). In the early days after injury, the growth factor is released from platelets and endothelial cells. It serves as a chemotactic signal for neutrophils, macrophages, and fibroblasts to the damaged tissue. Continued stimulation of these cells after their arrival to the wound leads to endogenous production of PDGF, provisional ECM synthesis, fibroblast proliferation, and collagen production. After 2-3 weeks, PDGF signaling contributes to wound remodeling by orchestrating active collagen turnover and cross-linking. The temporal changes in the effects of PDGF appear to be determined in part by its relative concentration

\begin{tabular}{|c|c|c|c|}
\hline Time & $\begin{array}{l}\text { Stages of } \\
\text { wound healing }\end{array}$ & $\begin{array}{l}\text { Major sources } \\
\text { of PDGF }\end{array}$ & $\begin{array}{l}\text { Effects } \\
\text { of PDGF }\end{array}$ \\
\hline \multirow[t]{2}{*}{ Injury } & Hemostasis & \multirow[t]{2}{*}{$\begin{array}{l}\text { Platelets } \\
\text { endothelial cells }\end{array}$} & $\begin{array}{l}\text { Chemotaxis } \\
\text { of neutrophils, } \\
\text { macrophages, } \\
\text { fibroblasts }\end{array}$ \\
\hline & Inflammatory & & $\begin{array}{l}\text { Activation } \\
\text { of macrophages } \\
\text { \& fibroblasts } \\
\text { Endogenous GF } \\
\text { production } \\
\text { ECM synthesis }\end{array}$ \\
\hline 5-7 days & Proliferative & \multirow[t]{2}{*}{$\begin{array}{l}\text { Macrophages } \\
\text { fibroblasts }\end{array}$} & $\begin{array}{l}\text { Fibroblast proliferation } \\
\text { Collagen synthesis }\end{array}$ \\
\hline I year + & Remodeling & & $\begin{array}{l}\text { Collagenase expression } \\
\text { Active collagen turn- } \\
\text { over \& cross-linking }\end{array}$ \\
\hline
\end{tabular}

Figure 2 The cellular sources and effects of PDGF vary throughout the wound healing process.

Abbreviations: ECM, extracellular matrix; PDGF, platelet-derived growth factor. 
to other important growth factors such as TGF- $\beta$, insulin-like growth factor-1 (IGF-1), IL-1, epidermal growth factor (EGF), and TNF- $\alpha$ (Bennett et al 2003; Cross et al 2003).

As has been previously discussed, the care of a diabetic foot ulcer is not a minor endeavor. Many practitioners do not address all the disparate contributors (vascular, neuropathic, infectious, and biomechanical) to a foot ulcer. Poor compliance with wound care and off-loading remain a frustrating cause of therapeutic failures as well as recidivism. Biofilm recalcitrance can also become a frustrating aspect of ulcer care. All of these "real-world" practice considerations result in healing rates that do not match those seen in clinical trials; this is often not taken into account when evaluating efficacies of therapeutics such as becaplermin. It is also important to emphasize that the etiopathology of a diabetic foot ulcer does not result from a growth factor deficiency per se, but rather from a traumatic insult in the setting of peripheral neuropathy, with variable contributions from musculotendinous derangements, local and regional ischemia, and infection. Adding a growth factor to a wound without addressing these factors will be a futile exercise.

\section{Becaplermin as a therapeutic agent and evidence for clinical efficacy in diabetic forefoot ulcers}

Several important pre-clinical animal studies demonstrated the potential of PDGF as a therapeutic agent for wound healing. These studies stemmed from the observations that most of the cell types entering the wound are capable of synthesizing PDGF and are also activated by PDGF and the hypothesis that exogenous application of PDGF could amplify its proliferative effect through an autocrine feedback loop (Pierce et al 1991). One study utilizing a rat incisional wound model showed increased inflammatory response, wound cellularity, granulation tissue formation, neovascularization, and epithelialization in PDGF-treated wounds the over control wounds. Along with those parameters, an accelerated wound healing rate and increased wound breaking strength were also shown (Pierce et al 1988). Another study performed in a rabbit ear excisional wound model revealed significantly increased re-epithelialization and granulation tissue formation in the PDGF-treated wounds (Mustoe et al 1991). Furthermore, studies of impaired wound healing in a diabetic mouse strain demonstrated a reversal of the deficient healing phenotype with PDGF treatment (Greenhalgh et al 1990).

These successful animal studies led to human clinical trials investigating the potential of exogenous PDGF therapy for healing chronic wounds. Initial trials were carried out in the setting of pressure sores and suggested that PDGF treatment had therapeutic value in the acceleration of chronic wound healing. However, continued studies in this patient population did not appear to bear out these findings (Mustoe et al 1994). Other trials, which focused on diabetic foot ulcers, produced statistically significant findings that eventually led to FDA approval for use in this subset of chronic wounds (Steed 1995; d'Hemecourt et al 1998; Wieman et al 1998; Smiell et al 1999).

When it was approved by the FDA in 1997 for the treatment of chronic lower extremity diabetic neuropathic ulcers, rhPDGF-BB became the first and remains the only pharmacological agent approved for treatment of a chronic wound condition. This recombinant growth factor is delivered in a topical aqueous-based sodium carboxymethylcellulose ( $\mathrm{NaCMC}$ ) gel and is marketed as Regranex ${ }^{\circledR}$. The therapy is indicated for uninfected diabetic foot ulcers that extend into the subcutaneous tissue and have an adequate vascular supply.

The efficacy of becaplermin gel treatment was evaluated in 4 published multicenter prospective randomized control trials (RCTs), which led to its FDA approval. Initially headed by Steed and the Diabetic Ulcer Study Group, these trials involved a total of 922 patients. The first two studies were double-blinded, while the third and fourth studies were only evaluator-blinded because they involved an arm with only standard ulcer care. Ulcer criteria for entrance into each of these trials included the following: wound presence for greater than 8 weeks, absence of infection, presence of an adequate vascular supply, and presence of good wound care. Ulcer sizes were restricted to those between $1 \mathrm{~cm}^{2}$ and 100 $\mathrm{cm}^{2}$, but only the first trial included those larger than $40 \mathrm{~cm}^{2}$, and over $95 \%$ of the wounds had areas of less than $10 \mathrm{~cm}^{2}$. All of the wounds were sharply debrided to obtain a clean wound bed before randomization; however, one limitation of these trials was the variable debridement regimens that followed. The treatment protocol for the application of becaplermin gel and dressing changes remained consistent throughout the studies. On the other hand, patient adherence to important aspects of diabetic ulcer care such as off-loading appeared to be variable. The trials all continued for 20 weeks to evaluate complete wound closure as the primary endpoint, but the only reported follow-up after the conclusion of each study was a 3-month period. The results were analyzed using multiple statistical methods that were appropriate for the data presented. Becaplermin appeared to have an acceptable safety profile, was well-tolerated by the patients, and could easily be used by the patients or their caregivers (Steed 1995; d'Hemecourt et al 1998; Wieman et al 1998; Smiell et al 1999; Steed 2006). 
The first study was a multicenter double-blinded RCT that compared becaplermin gel $(30 \mu \mathrm{g} / \mathrm{g})$ against a placebo gel (NaCMC) for clinical efficacy and safety (Steed 1995). This Phase II trial was the first to investigate PDGF in human diabetic ulcers and recruited 118 patients. The authors reported a statistically significant greater incidence of complete healing in the treated wounds ( $48 \%$ vs $25 \%, \mathrm{p}=0.02)$.

A subsequent Phase III trial assigned 382 patients to becaplermin gel $(30 \mu \mathrm{g} / \mathrm{g}$ and $100 \mu \mathrm{g} / \mathrm{g})$ and placebo gel treatment groups (Wieman et al 1998). The becaplermintreated wounds had higher incidences of complete healing over the study period, but only the $100 \mu \mathrm{g} / \mathrm{g}$ becaplermin dose yielded statistically significant results when compared to placebo gel $(50 \%$ vs $35 \%, \mathrm{p}=0.01)$.

The third smaller study randomized a total of 138 patients to placebo gel treatment and good ulcer care comparison groups, in addition to a smaller group of 34 patients who opted to receive active becaplermin gel treatment $(100 \mu \mathrm{g} / \mathrm{g})$ instead (d'Hemecourt et al 1998). The becaplermin group was apparently not powered for statistical comparison of wound healing because the primary goal of this trial was to compare the safety of the placebo gel group against good ulcer care. The complete wound healing incidences were reported to be $44 \%$ for becaplermin gel treatment, $36 \%$ for placebo gel treatment, and $22 \%$ for good ulcer care only. However, these outcomes were not statistically significant at the $\mathrm{p} \leq 0.05$ level.

A fourth trial of 250 patients was designed to assess resource utilization by comparing becaplermin gel treatment $(100 \mu \mathrm{g} / \mathrm{g})$ against good ulcer care alone. The study did not demonstrate a statistically significant difference in complete wound healing incidences between the two groups ( $36 \%$ vs $32 \%$ ), although the authors did note that a trend in favor of becaplermin gel treatment appeared to be present (Smiell et al 1999).

An additional post-marketing Phase IV trial was performed with the goal to demonstrate becaplermin gel treatment efficacy against that of good ulcer care (Robson et al 2005). The main difference in the treatment design of this trial as opposed to the four previous pre-marketing RCTs was that this study utilized a once-daily application instead of a twice-daily application of becaplermin gel. The study was also limited by the fact that it did not achieve full accrual of patients. Therefore, it was not appropriately powered for statistical comparison of complete wound healing incidences. For the 146 out of the 340 planned patients, the reported complete healing incidences were $42 \%$ for becaplermin gel treatment and $35 \%$ for good ulcer care $(\mathrm{p}=0.316)$.

Recent meta-analyses have been performed to reconcile the data from these RCTs (Smiell et al 1999). A logistic regression model showed that for a median wound size of $1.5 \mathrm{~cm}^{2}$, the probability of complete healing was increased $39 \%$ with the administration of becaplermin gel $(100 \mu \mathrm{g} / \mathrm{g})$ over placebo gel alone ( $50 \%$ vs $36 \%, \mathrm{p}=0.007)$. Similar results were found when comparing becaplermin gel treatment to only good ulcer care. Analysis of the data using a Cox proportional hazards model suggested that becaplermin gel $(100 \mu \mathrm{g} / \mathrm{g})$ treatment also significantly decreased the time to achieve complete wound healing in comparison to placebo gel treatment $(\mathrm{p}=0.01)$. Using the Kaplan-Meier method, this difference in healing time was estimated to be $30 \%$ less for becaplermin gel at the 35 th percentile level (14.1 weeks vs 20.1 weeks). Robson et al also performed a meta-analysis after the Phase IV trial to integrate those results with the previous RCTs. While the Phase IV study itself did not reveal statistically significant results, the overall meta-analysis results did not deviate remarkably from that of the first four RCTs.

Some important issues in studying human wound healing therapeutics have been highlighted by these trials. In this regard they can be considered bellwether studies in the field of wound care. The baseline variability of wound healing is particularly notable and is illustrated in these studies as the wound closure rates for the placebo gel treatment and standard care groups ranged from $23 \%$ to $36 \%$. Similarly, the becaplermin treatment groups also had wound closure rates that varied from $36 \%$ to $50 \%$. With such variability, trials enrolling as many as 200 patients may still be underpowered to demonstrate a treatment effect (Mustoe 2006). The durability of the treatment effect is another consideration of such wound healing studies and has implications for the cost-effectiveness of the therapy. In these becaplermin trials, the follow-up included a 3-month period after the 20-week study period ended. The recurrence rate was found to be $28 \%$, regardless of the treatment group. Importantly, there was not a standardized regimen of ulcer prevention strategies implemented during this three month period. While recurrence may have likely been primarily dependent on patient compliance to continued wound and foot care strategies, it remains a concern when extrapolating these trial results to routine clinical practice.

While the ability to demonstrate a treatment effect with becaplermin represents a giant step forward in the treatment of chronic wounds, many concerns about the management of diabetic foot ulcers with becaplermin remain to be answered. The becaplermin trials only studied diabetic forefoot ulcers, which are typically smaller and less prone to ischemia than midfoot or hindfoot ulcers. Comparison of becaplermin 
gel to other modalities, such as the living bilayered construct, Apligraf ${ }^{\circledR}$ (Organogenesis, Inc., Canton, MA), has not been performed. These studies also did not compare becaplermin therapy to a rigorous off-loading regimen, which has actually been shown to heal a large proportion of foot ulcers (Wu et al 2006). Other variables such as patient co-morbidities, osteomyelitis, biofilm, and debridement regimens will certainly remain challenges for assessing the use of becaplermin. Nevertheless, in the appropriate clinical scenarios, the utilization of becaplermin gel as an adjunct to diabetic foot ulcer care can lead to improved results.

\section{Economic analyses of becaplermin therapy}

The burdensome strains of diabetic complications exist on personal, social, medical, and economical levels. Depending on the viewpoints that are considered, the costs and consequences can vary dramatically. While personal losses such as pain and suffering are difficult to quantify, multiple analyses have been conducted to study the financial impact of diabetes on the national health care system, the economy, and the society. Direct health care costs in the United States for diabetes are estimated to be over US\$90 billion annually, consuming approximately $10 \%$ of national health care expenditures. One-third of this spending is directed to the care of diabetic complications. Another US\$42 billion of indirect costs result from lost productivity, disability, and mortality (Centers for Disease Control and Prevention 2005).

A logical question has focused on the cost-effectiveness of implementing becaplermin therapy. Although raising the initial costs, the benefits in the long run potentially result in overall cost savings. Sequelae such as infection and gangrene result in additional hospitalizations and possible amputations, adding significant morbidity and cost to the care of diabetic foot ulcers. By improving the treatment efficacy of neuropathic ulcers, becaplermin would likely reduce the risk for the development of lower extremity infections and gangrene. In summary, any modality that improves overall wound healing, and prevents additional hospitalization and amputation, would likely be cost-effective in monetary terms and certainly efficacious in reducing patient morbidity.

With the caveat that economic analyses of clinical scenarios inherently contain many limitations, those examining the treatment of diabetic foot ulcers have suggested that becaplermin in conjunction with good wound care may be cost-effective (Table 2). In a study published in 2001, Kantor and Margolis (2001) estimated the cost-effectiveness of becaplermin therapy in comparison to the modalities of standard care, specialized care at a wound care center, and treatment with platelet releasate. They performed a deterministic analysis by utilizing data from published sources. Conducted from the viewpoint of the payer, the study used Medicare data to proxy for costs, thereby not considering indirect costs. Effectiveness data were derived from RCTs and meta-analyses focusing on treatments for neuropathic ulcers, including those investigating becaplermin. For their analyses, effectiveness was defined in terms of percentage of the wound healed at 20 weeks and 32 weeks. The study period was limited to the length of the clinical trials. Incremental cost-effectiveness ratios that were computed suggested that becaplermin therapy had the most favorable marginal costs associated with each additional percentage increase in healing at 20 weeks.

Given the potential impact of becaplermin therapy beyond the treatment period, Persson et al (2000) attempted to extrapolate their data to 1- and 2-year time points by developing a Markov economic model to simulate the management of diabetic foot ulcers. This approach is useful for studying clinical problems that involves a continuous risk, events that may occur multiple times, and outcome utilities that depend on the timing of different events. The costs were examined from a payer's viewpoint in the Swedish health care system and were based on a cohort study of 314 Swedish patients. The efficacy of good wound care was based on a 9 month follow-up study on 194 diabetic patients in the United States with neuropathic foot

Table 2 Recent cost-effectiveness studies of becaplermin usage in diabetic foot ulcers have suggested an overall cost savings for their use

\begin{tabular}{|c|c|c|}
\hline Study & Type & Conclusions \\
\hline $\begin{array}{l}\text { Kantor and } \\
\text { Margolis (200I) }\end{array}$ & $\begin{array}{l}\text { Deterministic } \\
\text { analysis }\end{array}$ & $\begin{array}{l}\text { Compared becaplermin to stan- } \\
\text { dard care, care at wound center, } \\
\text { and platelet releasate treatment } \\
\text { Becaplermin had the most } \\
\text { favorable marginal costs associ } \\
\text { ated with each percentage } \\
\text { increase of healing }\end{array}$ \\
\hline $\begin{array}{l}\text { Persson et al } \\
(2000)\end{array}$ & $\begin{array}{l}\text { Markov } \\
\text { model }\end{array}$ & $\begin{array}{l}\text { Compared becaplermin to stan- } \\
\text { dard care Becaplermin treatment } \\
\text { resulted in a } 24 \% \text { increase of } \\
\text { ulcer-free months and a } 9 \% \\
\text { decrease in the amputation rate } \\
\text { over a } 2 \text {-year period, while } \\
\text { consuming less overall resources }\end{array}$ \\
\hline $\begin{array}{l}\text { Sibbald et al } \\
(2003)\end{array}$ & $\begin{array}{l}\text { Decision analytic } \\
\text { model }\end{array}$ & $\begin{array}{l}\text { Compared becaplermin to stan- } \\
\text { dard care Incorporation of beca } \\
\text { plermin treatment with standard } \\
\text { care resulted in } 26 \text { fewer ulcer- } \\
\text { days over I year }\end{array}$ \\
\hline
\end{tabular}


ulcers. Becaplermin treatment efficacy was derived from the four major RCTs. In contrast to the previous study, effectiveness was analyzed in terms of the number of ulcer-free months per year. Interestingly, their results suggested that becaplermin therapy with good wound care was actually a win-win situation, in which becaplermin treatment both consumed less overall resources and generated better outcomes than standard wound care alone (Sonnenberg et al 1993; Drummond et al 1997; O’Brien et al 1997; Sibbald et al 2003).

Other approaches of evaluating cost-effectiveness include quality of life (QOL) studies, despite the increased difficulty in quantifying the relevant variables. Recent studies employing this method have suggested that the QOL scores between patients who have suffered lower extremity amputations due to trauma and those who have been reconstructed were actually similar. However, the diabetic patient population should be distinguished from these patients. The increased cardiovascular morbidity from an amputation in a diabetic patient is significant, and the avoidance of this outcome warrants intensive efforts at foot salvage (Sibbald et al 2003).

\section{Recommendations for becaplermin utilization}

The current management paradigm of diabetic neuropathic foot ulcers involves maximizing the standard treatment strategies discussed earlier. For the majority of non-ischemic and uninfected wounds, this treatment regimen should result in complete healing. These principles echo guidelines published by organizations such as the American Diabetes Association, the College of Foot and Ankle Surgeons, the International Working Group on the Diabetic Foot, and the Wound Healing Society (Apelqvist et al 2000; Brem et al 2006; Steed et al 2006; Rathur et al 2007).

Most authorities recommend utilizing becaplermin as an adjunct therapy for those foot ulcers that do not respond acceptably to optimized standard strategies. For example, Steed et al (2006) considers becaplermin therapy for any patient with a diabetic foot ulcer in which standard care does not result in wound reduction by at least $40 \%$ in 4 weeks, and we would endorse his recommendations (Figure 3). However, the utility of employing becaplermin therapy earlier in those patients with wounds at high risk for failure, such as large foot ulcers, or employing becaplermin therapy in patients with uncomplicated wounds remains unclear (Wieman 2005). In the case of aged patients ( $>70$ years), we will more readily add becaplermin to ulcer therapy, as there are likely more senescent cells and a relative growth factor deficiency that may derive more benefit from growth factor supplementation
(Mustoe et al 2006). Clinical data to support this use are currently being gathered.

\section{Conclusion}

Although becaplermin is currently only approved for use in neuropathic diabetic foot ulcers, the potential benefit of PDGF therapy may still exist in other difficult chronic wounds. For pressure sores, the early clinical trials unfortunately did not appear to bear promising results. However, wounds that develop in patients who are undergoing antiangiogenic treatment, are receiving radiation therapy, or have both diabetes and renal failure remain problematic and may benefit from PDGF therapy.

New forms of growth factor therapy may yet be able to modulate the chronic wound healing process to a greater degree than becaplermin therapy today. Ongoing investigations are studying the combination of growth factors, the improvement of growth factor vehicles, the utilization of tissue engineering constructs with growth factors, the interaction of stem cells in conjunction with growth factors, and gene therapy modalities. Already, some fruitful efforts have yielded a new generation in PDGF therapy, evidenced by FDA approval of GEM 21S (Growth-factor Enhanced Matrix, rhPDGF-BB, OsteoHealth Company, Shirley, NY) last year for the treatment of periodontal bone defects and associated gingival recession. This new therapeutic combines rhPDGF$\mathrm{BB}$ and a synthetic bone matrix to stimulate new bone growth while providing a scaffold for that growth to occur.

Nevertheless, it has become clear that new treatment strategies for chronic wounds will need to address multiple factors simultaneously, instead of just one aspect of wound healing. Future successful therapeutics for diabetic neuropathic ulcers will likely parallel the development of effective therapies for chronic wounds as a whole. A new generation of growth factor therapy may very well be on the horizon for these wounds, but such therapeutics will likely need to be integrated into a multifaceted regimen to be most effective.

The management of diabetic neuropathic foot ulcers continues to be a frustrating problem for all clinicians. Not only is the effectiveness of therapy oftentimes inadequate, but the costs of treatment continue to soar. For the wounds that fail to heal acceptably despite optimized standard care, becaplermin gel can potentially bridge the gap to complete healing. As the first, and to date only, growth factor approved for the treatment of diabetic foot ulcers, becaplermin has broken new ground and has set the standard against which all future modalities will be compared. Not trivially, much has been learned from the pivotal becaplermin trials about the execution of successful 


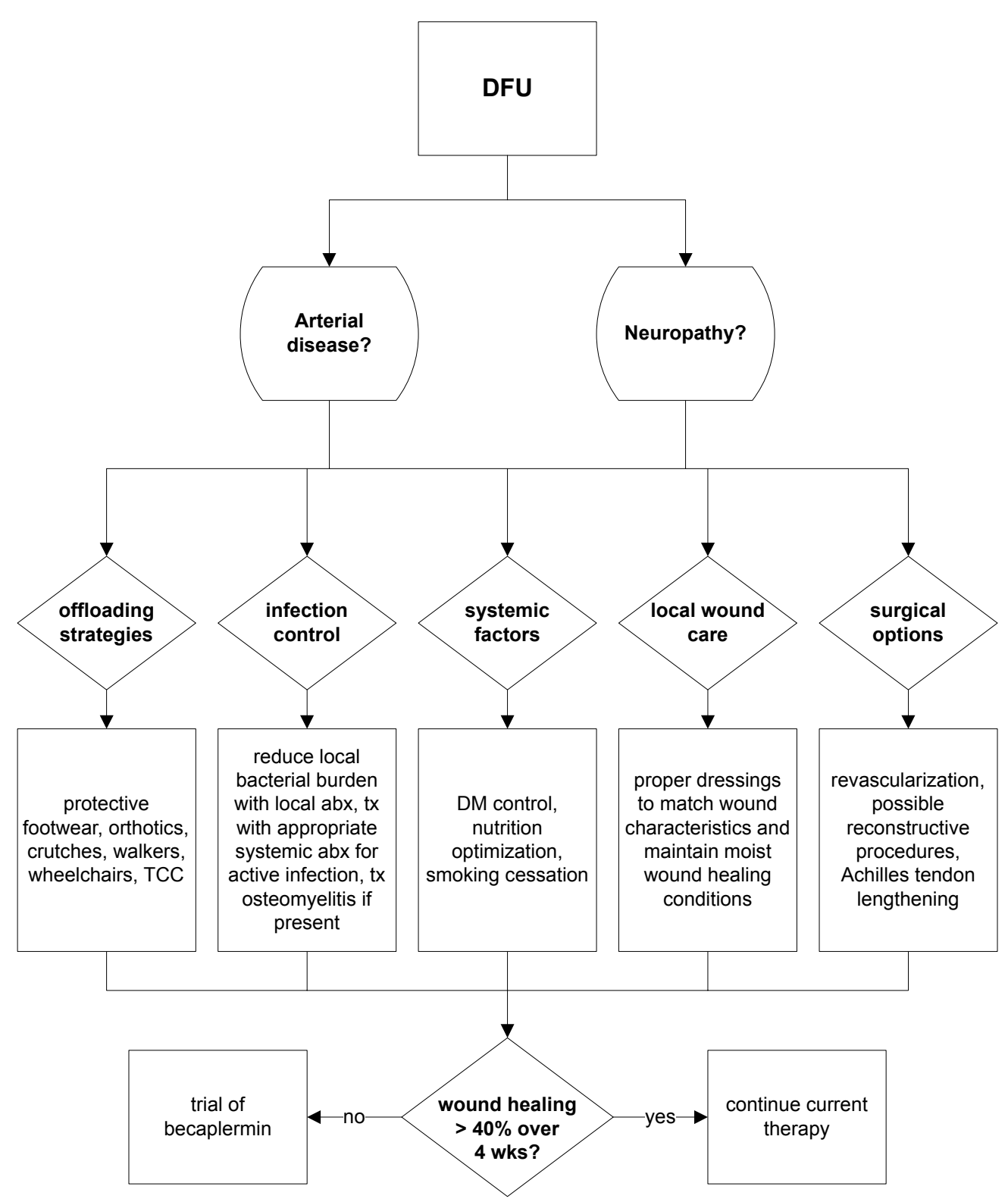

Figure 3 The algorithm for diabetic foot ulcer care practiced by Northwestern Memorial Hospital plastic surgeons follows closely with guidelines published by the Wound Healing Society.

clinical investigations. These insights can be applied directly to the investigation of future modalities in the difficult arena of wound care. It still remains unclear at this time if the effectiveness of becaplermin is significant enough to warrant more liberal use. In the future, it appears hopeful that a new generation of growth factor therapy will follow becaplermin for the treatment these chronic wounds.

\section{References}

Apelqvist J, Bakker K, van Houtum WH, et al. 2000. International consensus and practical guidelines on the management and the prevention of the diabetic foot. International Working Group on the Diabetic Foot. Diabetes Metab Res Rev, 16(Suppl 1); S84-S92.
Attinger CE, Ducic I, Cooper P, et al. 2002. The role of intrinsic muscle flaps of the foot for bone coverage in foot and ankle defects in diabetic and nondiabetic patients. Plast Reconstr Surg, 110:1047-54.

Bennett NT, Schultz GS. 1993. Growth factors and wound healing: Part II. Role in normal and chronic wound healing. Am J Surg, 166:74-81.

Bennett SP, Griffiths GD, Schor AM, et al. 2003. Growth factors in the treatment of diabetic foot ulcers. Br J Surg, 90:133-46.

Blazer S, Khankin E, Segev Y, et al. 2002. High glucose-induced replicative senescence: point of no return and effect of telomerase. Biochem Biophys Res Commun, 296:93-101.

Boulton AJ, Kirsner RS, Vileikyte L. 2004. Clinical practice. Neuropathic diabetic foot ulcers. $N$ Engl J Med, 351:48-55.

Brem H, Sheehan P, Rosenberg HJ, et al. 2006. Evidence-based protocol for diabetic foot ulcers. Plast Reconstr Surg, 117(7 Suppl):193S-209S.

Brownlee M. 2005. The pathobiology of diabetic complications: a unifying mechanism. Diabetes, 54:1615-25. 
Cavanagh PR, Lipsky BA, Bradbury AW, et al. 2005. Treatment for diabetic foot ulcers. Lancet, 366:1725-35.

Centers for Disease Control and Prevention. 2005. National diabetes fact sheet: general information and national estimates on diabetes in the United States.

Chaudhry V, Stevens JC, Kincaid J, et al. 2006. Practice Advisory: utility of surgical decompression for treatment of diabetic neuropathy: report of the Therapeutics and Technology Assessment Subcommittee of the American Academy of Neurology. Neurology, 66:1805-8.

Cowie CC, Rust KF, Byrd-Holt DD, et al. 2006, Prevalence of diabetes and impaired fasting glucose in adults in the U.S. population: National Health And Nutrition Examination Survey 1999-2002. Diabetes Care, 29:1263-8.

Cross KJ, Mustoe TA. 2003.Growth factors in wound healing. Surg Clin North Am, 83531-45, vi.

d'Hemecourt PA, Smiell JM, Karim MR. 1998. Sodium carboxymethylcellulose aqueous-based gel vs. becaplermin gel in patients with nonhealing lower extremity diabetic ulcers. Wounds, 10:69-75.

Drummond MF, Richardson WS, O'Brien BJ, et al. 1997. Users' guides to the medical literature. XIII. How to use an article on economic analysis of clinical practice. A. Are the results of the study valid? EvidenceBased Medicine Working Group. JAMA, 277:1552-7.

Engerman RL, Kern TS, Larson ME. 1994. Nerve conduction and aldose reductase inhibition during 5 years of diabetes or galactosaemia in dogs. Diabetologia, 37:141-4.

Falanga, V. 2005. Wound healing and its impairment in the diabetic foot. Lancet, 366:1736-43.

Frykberg RG, Zgonis T, Armstrong DG, et al. 2006. Diabetic foot disorders. A clinical practice guideline (2006 revision). J Foot Ankle Surg, 45(5 Suppl):S1-66.

Gabbay KH, Merola LO, Field RA. 1966. Sorbitol pathway: presence in nerve and cord with substrate accumulation in diabetes. Science, 151:209-10.

Greenhalgh DG, Sprugel KH, Murray MJ, et al. 1990. PDGF and FGF stimulate wound healing in the genetically diabetic mouse. Am J Pathol, 136:1235-46.

Hammes HP, Martin S, Federlin K, et al. 1991. Aminoguanidine treatment inhibits the development of experimental diabetic retinopathy. Proc Natl Acad Sci USA, 88:11555-8.

Ishii H, Jirousek MR, Koya D, et al. 1996. Amelioration of vascular dysfunctions in diabetic rats by an oral PKC beta inhibitor. Science, 272:728-31.

Jaap AJ, Shore AC, Stockman AJ, et al. 1996. Skin capillary density in subjects with impaired glucose tolerance and patients with type 2 diabetes. Diabet Med, 13:160-4.

Kantor J, Margolis DJ. 2001. Treatment options for diabetic neuropathic foot ulcers: a cost-effectiveness analysis. Dermatol Surg, 27:347-51.

Koya D, King GL. 1998. Protein kinase C activation and the development of diabetic complications. Diabetes, 47:859-66.

LoGerfo FW, Coffman JD. 1984. Current concepts. Vascular and microvascular disease of the foot in diabetes. Implications for foot care. N Engl J Med, 311:1615-19.

Martin A, Komada MR, Sane DC. 2003. Abnormal angiogenesis in diabetes mellitus. Med Res Rev, 23:117-45.

Medina A, Scott PG, Ghahary A, et al. 2005. Pathophysiology of chronic nonhealing wounds. J Burn Care Rehabil, 26:306-19.

Mueller MJ, Sinacore DR, Hastings MK, et al. 2003. Effect of Achilles tendon lengthening on neuropathic plantar ulcers. A randomized clinical trial. J Bone Joint Surg Am, 85-A:1436-45.

Mustoe TA. 2006. Discussion: clinical evaluation of recombinant human platelet-derived growth factor for the treatment of lower extremity ulcers. Plast Reconstr Surg, 117(7 Suppl):150S-1S.

Mustoe TA, Cutler NR, Allman RM, et al. 1994. A phase II study to evaluate recombinant platelet-derived growth factor-BB in the treatment of stage 3 and 4 pressure ulcers. Arch Surg, 129:213-19.

Mustoe TA, O'Shaughnessy K, Kloeters O. 2006, Chronic wound pathogenesis and current treatment strategies: a unifying hypothesis. Plast Reconstr Surg, 117(7 Suppl):35S-41S.
Mustoe TA, Pierce GF, Morishima C, et al. 1991. Growth factor-induced acceleration of tissue repair through direct and inductive activities in a rabbit dermal ulcer model. J Clin Invest, 87:694-703.

O'Brien BJ, Heyland D, Richardson WS, et al. 1997. Users' guides to the medical literature. XIII. How to use an article on economic analysis of clinical practice. B. What are the results and will they help me in caring for my patients? Evidence-Based Medicine Working Group. JAMA, 277:1802-6.

Persson U, Willis M, Odegaard K, et al. 2000. The cost-effectiveness of treating diabetic lower extremity ulcers with becaplermin (Regranex): a core model with an application using Swedish cost data. Value Health, 3(Suppl 1):39-46.

Pierce GF, Mustoe TA, Altrock BW, et al. 1991. Role of platelet-derived growth factor in wound healing. $J$ Cell Biochem, 45:319-26.

Pierce GF, Mustoe TA, Senior RM, et al. 1988. In vivo incisional wound healing augmented by platelet-derived growth factor and recombinant c-sis gene homodimeric proteins. J Exp Med, 167:974-87.

Pinzur MS, Gold J, Schwartz D, et al. 1992. Energy demands for walking in dysvascular amputees as related to the level of amputation. Orthopedics, 15:1033-6.

Rathur HM, Boulton AJ. 2007. The neuropathic diabetic foot. Nat Clin Pract Endocrinol Metab, 3:14-25.

Rayman G, Malik RA, Sharma AK, et al. 1995. Microvascular response to tissue injury and capillary ultrastructure in the foot skin of type I diabetic patients. Clin Sci (Lond), 89:467-74.

Rayman G, Williams SA, Spencer PD, et al. 1986. Impaired microvascular hyperaemic response to minor skin trauma in type I diabetes. $\mathrm{Br} \mathrm{Med}$ $J$ (Clin Res Ed), 292:1295-8.

Robson MC, Mustoe TA, Hunt TK. 1998. The future of recombinant growth factors in wound healing. Am J Surg, 176(2A Suppl):80S-2S.

Robson M, Payne WG, Garner WL, et al. 2005. Integrating the results of phase IV (postmarketing) clinical trial with four previous trials reinforces the position that Regranex (becaplermin) gel $0.01 \%$ is an effective adjunct to the treatment of diabetic foot ulcers. $J$ Appl Res, 5:35-45.

Ross R, Glomset J, Kariya B, et al. 1974. A platelet-dependent serum factor that stimulates the proliferation of arterial smooth muscle cells in vitro. Proc Natl Acad Sci USA, 71:1207-10.

Schmidt AM, Hori O, Chen JX, et al. 1995, Advanced glycation endproducts interacting with their endothelial receptor induce expression of vascular cell adhesion molecule-1 (VCAM-1) in cultured human endothelial cells and in mice. A potential mechanism for the accelerated vasculopathy of diabetes, $J$ Clin Invest, 96, no. 3:1395-1403.

Sibbald RG, Torrance G, Hux M, et al. 2003. Cost-effectiveness of becaplermin for nonhealing neuropathic diabetic foot ulcers. Ostomy Wound Manage, 49:76-84.

Smiell JM, Wieman TJ, Steed DL, et al. 1999. Efficacy and safety of becaplermin (recombinant human platelet-derived growth factorBB) in patients with nonhealing, lower extremity diabetic ulcers: a combined analysis of four randomized studies. Wound Repair Regen, 7:335-46.

Sonnenberg FA, Beck JR. 1993. Markov models in medical decision making: a practical guide. Med Decis Making, 13:322-38.

Steed DL. 1995. Clinical evaluation of recombinant human platelet-derived growth factor for the treatment of lower extremity diabetic ulcers. Diabetic Ulcer Study Group. J Vasc Surg, 21:71-8.

Steed DL. 2006. Clinical evaluation of recombinant human platelet-derived growth factor for the treatment of lower extremity ulcers. Plast Reconstr Surg, 117(7 Suppl):143S-9S.

Steed DL, Attinger C, Colaizzi T, et al. 2006. Guidelines for the treatment of diabetic ulcers. Wound Repair Regen, 14:680-92.

Stone PA, Back MR, Armstrong PA, et al. 2005. Midfoot amputations expand limb salvage rates for diabetic foot infections. Ann Vasc Surg, 19:805-11.

Valdivia JM, Dellon AL, Weinand ME, et al. 2005. Surgical treatment of peripheral neuropathy: outcomes from 100 consecutive decompressions. J Am Podiatr Med Assoc, 95:451-4. 
Waters RL, Perry J, Antonelli D, et al. 1976. Energy cost of walking of amputees: the influence of level of amputation. J Bone Joint Surg Am, 58:42-6.

Wieman TJ. 2005. Principles of management: the diabetic foot. Am J Surg, 190:295-9.

Wieman TJ, Smiell JM, Su Y. 1998. Efficacy and safety of a topical gel formulation of recombinant human platelet-derived growth factor-BB (becaplermin) in patients with chronic neuropathic diabetic ulcers. A phase III randomized placebo-controlled double-blind study. Diabetes Care, 21:822-7.

Wu SC, Armstrong DG. 2006. The role of activity, adherence, and offloading on the healing of diabetic foot wounds. Plast Reconstr Surg, 117(7 Suppl):248S-53S. 